\title{
Enhancement of the Purcell factor in multiperiodic hyperboliclike metamaterials
}

\author{
Chebykin, A. V.; Babicheva, V. E.; Iorsh, I. V.; Orlov, A. A.; Belov, P. A.; Zhukovsky, Sergei
}

Published in:

Physical Review A

Link to article, DOI:

10.1103/PhysRevA.93.033855

Publication date:

2016

Document Version

Publisher's PDF, also known as Version of record

Link back to DTU Orbit

Citation (APA):

Chebykin, A. V., Babicheva, V. E., lorsh, I. V., Orlov, A. A., Belov, P. A., \& Zhukovsky, S. (2016). Enhancement of the Purcell factor in multiperiodic hyperboliclike metamaterials. Physical Review A, 93(3), [033855]. https://doi.org/10.1103/PhysRevA.93.033855

\section{General rights}

Copyright and moral rights for the publications made accessible in the public portal are retained by the authors and/or other copyright owners and it is a condition of accessing publications that users recognise and abide by the legal requirements associated with these rights.

- Users may download and print one copy of any publication from the public portal for the purpose of private study or research.

- You may not further distribute the material or use it for any profit-making activity or commercial gain

- You may freely distribute the URL identifying the publication in the public portal 


\title{
Enhancement of the Purcell factor in multiperiodic hyperboliclike metamaterials
}

\author{
A. V. Chebykin, ${ }^{1}$ V. E. Babicheva, ${ }^{2,1}$ I. V. Iorsh, ${ }^{1}$ A. A. Orlov,${ }^{1}$ P. A. Belov, ${ }^{1}$ and S. V. Zhukovsky ${ }^{1,3}$ \\ ${ }^{1}$ ITMO University, Kronverkskiy pr. 49, St. Petersburg 197101, Russia \\ ${ }^{2}$ Center for Nano-Optics, Georgia State University, P.O. Box 3965, Atlanta, Georgia 30302, USA \\ ${ }^{3}$ DTU Fotonik, Department of Photonics Engineering, Technical University of Denmark, Ørsteds Plads 343, DK-2800 Kgs. Lyngby, Denmark
}

(Received 23 June 2015; published 29 March 2016)

\begin{abstract}
Spontaneous emission enhancement is theoretically investigated in multiperiodic metal-dielectric multilayers (multiperiodic hyperboliclike metamaterials or photonic hypercrystals) where the unit cell consists of two layers of different dielectrics alternating with identical metallic layers. It is shown that the Purcell factor in such multiperiodic structures exceeds the Purcell factor in ordinary periodic hyperbolic or plasmonic metamaterials by a factor of 4 , which in general makes it possible to maximize interaction between emitting centers and nearby plasmonic structures. This enhancement is numerically characterized and shown to be related to the interplay between surface and volume plasmonic excitations in the multilayer metamaterial. We separately identify the influence of proximity between the emitter and the closest metal-dielectric boundary (including the quenching effect and the enhanced coupling of the dipole radiation and surface plasmon polaritons) and the effects related to the structural composition of the hypercrystal. The Purcell-factor modification brought about by placing a cavity layer into a multiperiodic structure was also characterized.
\end{abstract}

DOI: 10.1103/PhysRevA.93.033855

\section{INTRODUCTION}

Metamaterials, or artificially engineered nanocomposites, have become a very popular subject of research in the last decade, because these structures can provide electromagnetic properties that occur in natural materials rarely or not at all [1-3]. Within the concept of metamaterials one can realize negative refraction [4,5], giant optical activity [6], anomalous birefringence [7], ultrahigh Purcell-factor values [8,9], and optical axis precession dependent on the wave vector [10]. In particular, interest in such structures has increased dramatically with the progress in the field of nanostructure fabrication (see review [11] for more details and references).

Metamaterial realization of indefinite media, which are extremely anisotropic media with permittivity tensor components having different signs, are especially interesting for several reasons. On the one hand, these realizations, also called hyperbolic metamaterials (HMM) based on the fact that they feature hyperboloid-shaped isofrequency surfaces in their dispersion relation [11-16], enable propagation of waves with anomalously large wave vectors (arbitrarily large in the ideal case). Such "high- $k$ " waves, evanescent in conventional materials but propagating in hyperbolic metamaterials, are special because their wavelengths, equal to $2 \pi / k$, can be extremely short, so these waves can constitute subwavelength carriers of information. This property is applied in the idea of a hyperlens for image transmission with subwavelength resolution in the far field [17]. In addition, the existence of these high- $k$ waves leads to an increase of the density of photonic states in these structures, in turn leading to effects such as strong and broadband spontaneous emission enhancement $[8,18,19]$, reduction of the surface plasmon lasing threshold [20], slow light [21,22], enhancement of absorption [23] and nonlinearity [24,25], and anomalous heat transfer beyond the Stefan-Boltzmann limit [26-28].

On the other hand, hyperbolic metamaterials can be practically realized using relatively simple structures such as an array of nanorods embedded in the substrate material [29-32] or a metal-dielectric multilayer [33-35]. Propagation of high- $k$ waves in the hyperbolic medium has been clearly shown in [36,37]. As the characteristic size of the unit cell in hyperbolic metamaterials is much smaller than the wavelength of radiation in vacuum, one would expect that they can be described using the effective medium model, where the electromagnetic response is determined by the averaged properties of the composite rather than by the parameters of its individual elements, at least for wave vectors below the maximum values imposed by the finite size of the rods and layers $[9,18]$. However, recent studies showed that the effective medium approach in hyperbolic metamaterials breaks much sooner. The reason is that waves with large wave vectors propagating in the metamaterial in question have a wavelength much smaller than the wavelength in a vacuum, which modifies the limits of applicability of the effective medium model [38,39].

It was also shown that one can restore the validity of the effective medium approach by introducing nonlocal effective medium parameters [40]. However, whether it is through the nonlocal effects $[40,41]$ or through the photonic band-gap effects [38], it turns out that the geometric structure of a metamaterial's unit cell becomes very important in defining the metamaterial's optical properties. Therefore it becomes possible to control the propagation of high- $k$ waves in hyperbolic metamaterials by making the unit cell more complex. For example, in multilayer structures one can move from a simple metal-dielectric bilayer to a unit cell containing more layers. Such a change affects the effective medium parameters only weakly, but the metamaterial properties are found to change drastically. In a recent paper [42] we systematically studied the properties of such multiperiodic hyperbolic metamaterials with unit cells containing more than one type of metal or dielectric [see Fig. 1(a)]. We showed that multiperiodicity provides a way for engineering the metamaterial's response in the wave-vector space.

Such multiperiodic hyperbolic metamaterials can be regarded as periodic metamaterials with an additional level of structuring, which have been recently introduced as multiscale 
(a)

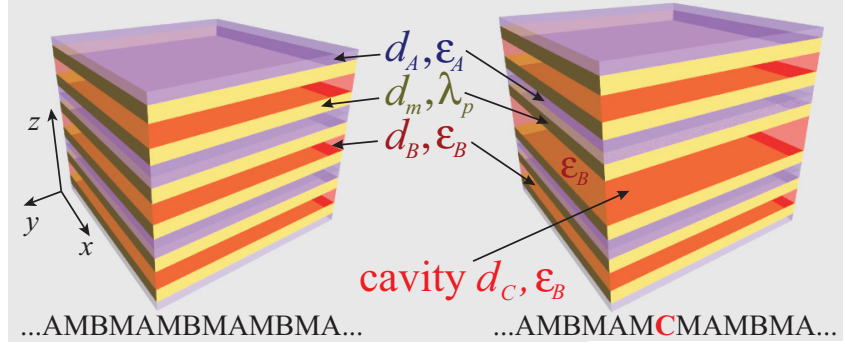

FIG. 1. Geometry of structures under consideration. The unit cell consists of four layers-two different dielectric (with thickness $d_{A}, d_{B}$ and dielectric permittivity $\varepsilon_{A}, \varepsilon_{B}$, denoted $\mathrm{A}$ and $\mathrm{B}$, respectively) and two similar metallic (denoted $\mathrm{M}$, with thickness $d_{m}$ and plasma wavelength $\lambda_{p}=c / v_{p}$ ). (a) Regular structure formed by repeating the unit cell MAMB over $N$ periods; (b) structure with the central layer having a different thickness $d_{C} \neq d_{B}$ forming a cavity layer $\mathrm{C}$.

hyperbolic metamaterials [43] or, slightly later, photonic hypercrystals [44,45]; the latter term suggests that a close relationship to photonic crystals makes it possible to transfer the well-studied photonic band-gap effects in complex multilayers [46] to plasmonic metamaterials. Specifically, it was shown that changing the metamaterial topology from periodic to multiperiodic and introducing photonic-crystallike features such as cavities [see Figs. 1(a) and 1(b)] can lead to unprecedented control both over bulk high- $k$ waves [43] and over surface waves [45]. It was also shown that multilayer structures support both resonant and nonresonant highpropagation wave vectors [47], and that resonant excitations are accompanied by slow energy propagation, which brings about an additional increase of the Purcell factor [48].

These results suggest that even though multilayered hyperbolic metamaterials have been rather well studied, there are many questions left to be answered once we depart from the simple alternating metal-dielectric stack in favor of a more complicated topology. One such question is the influence of the topology on the density of photonic states, and in turn, on spontaneous emission enhancement (Purcell factor) for an emitter embedded in a hyperbolic metamaterial. It was already determined that the Purcell enhancement can be very high for a simple metal-insulator-metal (MIM) structure with an ultrathin gap layer [47,49-51]; such enhancement is expected to persist when ultrathin constituent layers are present in a metamaterial setting. Moreover, the behavior of an emitter in a periodic metamaterial shows a strong dependence on the exact position of the emitter within a multilayer [9]. Therefore, studying the behavior of emitters in metamaterials with a complex multilayer topology is likely to reveal interesting effects, similar to the known strong density of states modification in photonic multilayers; this field has been a subject of avid research interest lately [1].

In this paper, we systematically characterize multiperiodic hyperbolic metamaterial structures and determine the Purcell factor for different placements of the emitter within them. Following the approach of [42], we focus on the biperiodic structure with the unit cell consisting of four layers: two layers of different dielectrics alternating with two similar metallic layers. We show that the dipole radiation of the emitter primarily couples to surface plasmon polaritons at the nearest metal-dielectric boundaries. However, the enhancement of the Purcell effect pertaining to the global geometrical properties of the structure is demonstrated and is apparently related to the interplay between surface and volume plasmonic excitations in the multilayer metamaterial. Hence, we show that multiperiodicity not only leads to spectral redistribution of the Purcell factor, but also enhances the spontaneous emission of an embedded dipole emitter beyond what is achievable in a regular periodic metal-dielectric structure. Therefore, increasing the complexity of the metamaterial topology is shown to be an alternative method of increasing the Purcell factor complementary to the known effect of decreasing the dielectric layer thicknesses previously demonstrated in MIM structures [52].

The paper is organized as follows. Section II is devoted to the description of the parameters of considered structures, as well as the problem formulation. In Sec. III we present the investigation of the Purcell factor in biperiodic hyperbolic metamaterials. We focus on the dependence of the Purcell factor on the permittivity of the dielectric layers in the structure, as well as on the emitter's position within the dielectric layers. Section IV describes the behavior of the Purcell factor in biperiodic structures with a cavity layer. Finally, Sec. V summarizes the paper.

\section{STRUCTURES AND METHODS}

Following the approach in [42], we define multiperiodic structures as metal-dielectric multilayers where the unit cell contains more than one type of plasmonic interface. One means to achieve this is to compose the unit cell of four layers denoted MBMA, where two layers made of a metal (denoted M, with thickness $d_{m}$ and permittivity $\varepsilon_{m}(\omega)$ varying according to the Drude model) alternate with two dielectric layers made of different dielectrics (denoted $\mathrm{A}$ and $\mathrm{B}$ with thicknesses $d_{A, B}$ and permittivities $\left.\varepsilon_{A, B}\right)$, as shown in Fig. 1(a). The structure's effective permittivity components can be determined using the standard quasistatic homogenization (see [11] for details):

$$
\begin{gathered}
\varepsilon_{\|}=\frac{\varepsilon_{A} d_{A}+\varepsilon_{B} d_{B}+2 \varepsilon_{m}(\omega) d_{m}}{d_{A}+d_{B}+2 d_{m}}, \\
\frac{1}{\varepsilon_{\perp}}=\frac{\varepsilon_{A}^{-1} d_{A}+\varepsilon_{B}^{-1} d_{B}+2 \varepsilon_{m}^{-1}(\omega) d_{m}}{d_{A}+d_{B}+2 d_{m}} .
\end{gathered}
$$

If $\varepsilon_{\|}$and $\varepsilon_{\perp}$ have different signs, we call such a structure a multiperiodic hyperbolic metamaterial. We note here that the term "hyperbolic" should be regarded only as a labeling term, since the effective medium approximation as used in Eqs. (1) and (2) is often inaccurate for complex plasmonic multilayers [53]. Moreover, in many cases a metamaterial that does not effectively have a hyperbolic dispersion (i.e., for which the product $\varepsilon_{\|} \varepsilon_{\perp}$ has a positive real part) still possesses many of the features that were originally attributed to hyperbolic metamaterials, such as the existence of high- $k$ plasmonic modes $[9,39]$ and strong spontaneous emission enhancement [54].

As opposed to the previous study [42] where considering infinite multiperiodic multilayers was necessary to determine 
their dispersion relations, we calculate the Purcell factor in finite-sized multilayers in order to bring the problem closer to a possible experimental realization; such a structure with $N$ periods can be symbolically represented as (MBMA) ${ }^{N}$. In addition to these structures, which we call regular since there are no geometrical features in them except multiperiodicity itself, we consider modified structures created by introducing a cavity (or defect), or in other words, by replacing one of the MBMA periods by MCMA, where $C$ denotes a cavity layer of thickness $d_{C}$ and permittivity $\varepsilon_{C}=\varepsilon_{B}$. The entire structure can then be coded as (MBMA) ${ }^{M}(\mathrm{MCMA})(\mathrm{MBMA})^{N-M-1}$. These structures are shown in Fig. 1(b).

Following the analysis in [43], we expect that the multiperiodicity will introduce a photonic band gap for high- $k$ waves, and adding a cavity layer will create a resonant state within that band gap and localize the energy of a bulk plasmonic wave. The goal of the present investigation is to find out whether, and to what extent, this modification of the high- $k$ wave-vector domain will affect spontaneous emission enhancement for an emitter embedded in various locations in the structure.

In order to do so, we numerically calculate the Purcell factor of an electric dipole emitter oriented along the $y$ axis and placed in a certain point $\mathbf{r}_{0}$. The calculation is based on the induced impedance numerical method [55], and in particular, on the relationship

$$
f=\frac{R_{\mathrm{in}}}{R_{\mathrm{in}}^{0}}=\frac{\operatorname{Im}\left[G_{y y}\left(\mathbf{r}_{0}, \mathbf{r}_{0}, \omega\right)\right]}{\operatorname{Im}\left[G_{y y}^{0}\left(\mathbf{r}_{0}, \mathbf{r}_{0}, \omega\right)\right]}=\frac{P_{\mathrm{rad}}}{P_{\mathrm{rad}}^{0}},
$$

where $P_{\text {rad }}, R_{\text {in }}$, and $G_{y y}$ are, respectively, the radiated power, the input impedance of the dipole, and the Green's function in the presence of the structure, whereas $P_{\mathrm{rad}}^{0}, R_{\mathrm{in}}^{0}$, and $G_{y y}^{0}$ are the same quantities in free space. Hence the Purcell factor $f=P_{\text {rad }} / P_{\text {rad }}^{0}$ can be derived from the input impedance of the dipole $R_{\text {in }}$, which is directly computed in the CST MICROWAVE STUDIO package [55].

This method was shown to be applicable to the Purcellfactor calculation in a variety of frequency ranges, i.e., from radio to optics $[55,56]$. More importantly, this method can be applied to structures with complex geometry and finite dimensions, where analytic approaches do not work.

The structures under study contain $N=10$ periods with each layer measuring $W=700 \mathrm{~nm}$ in $x$ and $y$ directions. The layer thicknesses are $d_{d}=d_{A}=d_{B}=37.5 \mathrm{~nm}$ and $d_{m}=$ $25 \mathrm{~nm}$. The permittivities of the dielectric layers $\varepsilon_{A}$ and $\varepsilon_{B}$ are varied, and for the metal layers the Drude model is assumed with equal to 83.4-THz collision frequency and varying plasma frequency $v_{p}$. A time-domain (transient) solver was used with the accuracy setting varied between -30 and $-40 \mathrm{~dB}$ to keep the numerical errors below $5 \%$. The boundary conditions are open (perfectly matched layers), with added space between the structure and the boundary. Additional symmetry planes with respect to the electric and magnetic fields are included to reduce the computational domain (electric symmetry on the $Y Z$ plane and magnetic symmetry on the $X Z$ plane). This way, a spatially finite structure in all directions is simulated. The lateral size of the structure $W$ was confirmed to be sufficiently large that the dipole placed at $x=y=0$ is not sensitive to the exact values of $W$, so that the chosen structure, while remaining practically relevant, enables direct comparison to the previously obtained results for the spatially infinite metamaterial.

\section{PURCELL EFFECT IN REGULAR MULTIPERIODIC STRUCTURES}

We first consider regular structures as shown in Fig. 1(a) and investigate the effect on the Purcell factor introduced by multiperiodicity. The point emitter is placed in the middle of the central layer, which is of type B (see inset in Fig. 2).

We first keep the permittivity of the dielectric layers $A$ constant $\left(\varepsilon_{A}=3.0\right)$ and vary the permittivity of dielectric in layers $B$, in one of which the emitter is located. Namely, $\varepsilon_{B}$ is set to vary from 1.8 to 4.6 ; the case $\varepsilon_{B}=3.0=\varepsilon_{A}$ corresponds to the conventional periodic hyperbolic metamaterials in the absence of multiperiodicity. The results are shown in Figs. 2(a) and 2(b). As one can see, even a minor degree of multiperiodicity $\left(\varepsilon_{B}=3.4\right)$ significantly modifies the Purcell factor, introducing two enhancement regions compared to only one region for $\varepsilon_{B}=3.0$. A further increase of $\varepsilon_{B}$ results in the shift of both enhancement regions towards longer wavelengths, with the lower-frequency enhancement region shifting more prominently, so that the gap between the two regions widens. Simultaneously, the peak value of the Purcell factor becomes much greater. Note that the Purcell-factor peak magnitude does not exhibit any marked preference with respect to $\varepsilon_{B}$ (where the emitter is placed), contrary to the expectation that it would be higher for an emitter placed in an optically denser environment; for instance, the Purcell factors for $\varepsilon_{B}=2.2$ and $\varepsilon_{B}=3.8$ are approximately the same on Figs. 2(a) and 2(b).

The same dependence but for fixed $\varepsilon_{B}=4.6$ and varying $\varepsilon_{A}$ from 3 to 5.8 is shown in Figs. 2(c) and 2(d); again, $\varepsilon_{A}=4.6=\varepsilon_{B}$ is the case of absent multiperiodicity. Similar to the previous case, we observe that multiperiodicity leads
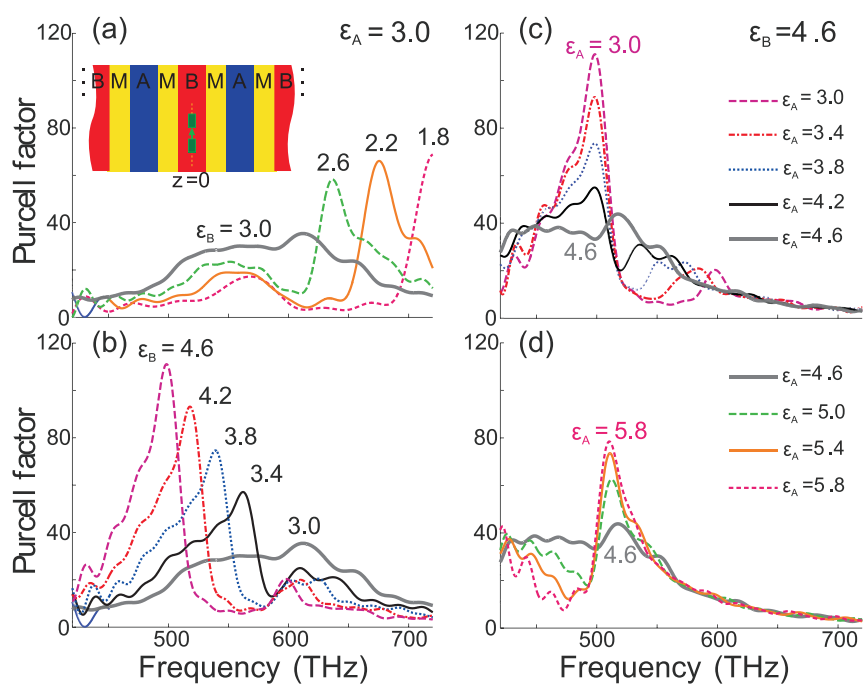

FIG. 2. Frequency dependence of the Purcell factor for the biperiodic structure shown in Fig. 1(a) for different layer permittivities: (a, b) for constant $\varepsilon_{A}=3$ and varying $\varepsilon_{B}$; (c, d) for varying $\varepsilon_{A}$ and constant $\varepsilon_{B}=4.6$. The point emitter is located in the middle of the central B layer ( $z=0$, see inset). The layer thicknesses are $d_{A}=d_{B}=37.5 \mathrm{~nm}$ and $d_{m}=25 \mathrm{~nm}$. Purcell factor is increased at frequencies that correspond to plasmon excitations. 


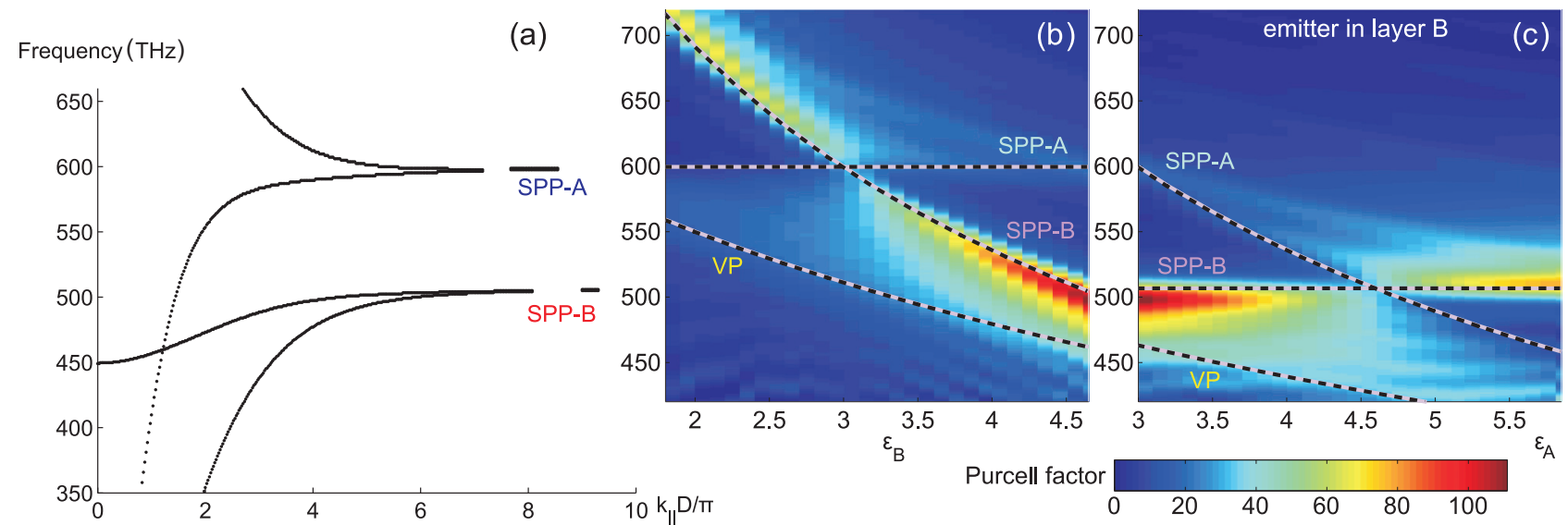

FIG. 3. (a) Dispersion diagram for bulk plasmon modes in a biperiodic structure shown in Fig. 1(a) with $\lambda_{p}=250 \mathrm{~nm}, \varepsilon_{A}=3$, and $\varepsilon_{B}=4.6$ in absence of losses [42]; for larger values of the in-plane projection of the wave vector $k_{\|}$the modes converge to the frequencies of the surface plasmons at the boundary between metal and $\varepsilon_{A}$ (SPP-A) or $\varepsilon_{B}$ (SPP-B), as given by Eq. (4); $k_{\|}$is normalized to the period $D=d_{A}+d_{B}+2 d_{m}$. (b) Frequency of the surface plasmon (SPP-A and SPP-B) resonances as well as the volume plasmon (VP) in a structure with constant $\varepsilon_{A}=3$ and varying $\varepsilon_{B}$, similar to Figs. 2(a) and 2(b), overlaid with the dependence of the Purcell factor on $\varepsilon_{B}$ and frequency. (c) Same as (b) but for the structure with varying $\varepsilon_{A}$ and constant $\varepsilon_{B}=4.6$, similar to Figs. 2(c) and 2(d). The emitter location is the same as in Fig. 2.

to a splitting of the region of the enhanced spontaneous emission into two regions separated by a gap. Since we are now modifying the permittivity of the layers that do not contain the emitter, the increase of the peak value of the Purcell factor as one decreases $\varepsilon_{A}$ from 4.6 to 3.0 can be attributed to the characteristic of the entire metamaterial rather than just the immediate vicinity of the emitter. For both cases in Fig. 2, we can thus observe that the multiperiodic hyperbolic metamaterials are able to enhance the spontaneous emission of an embedded dipole beyond ordinary hyperbolic metamaterials based on periodic multilayers.

To explain the observed changes in the Purcell factor, we analyze plasmonic excitations in the considered multilayer structure in more detail. Figure 3(a) shows an example dispersion relation of bulk plasmonic modes in the biperiodic structure with $\varepsilon_{A}=3, \varepsilon_{B}=4.6$, and calculations are done without taking metal losses into account for simplicity. These modes come from the hybridization of surface plasmon polariton (SPP) excitations [39] on multiple metal-dielectric interfaces. We see that for very large values of the tangential wave vector $k_{\|}$, the coupling between surface plasmons in different unit cells of the multilayer becomes strongly suppressed, and the bulk modes are seen to converge to the two SPP modes at individual M-A and M-B interfaces (labeled SPP-A and SPP-B, respectively), since there are two different plasmonic interfaces in the biperiodic structure [42]. The corresponding resonant frequencies $v_{s p A, s p B}$ are determined by

$$
v_{s p A, s p B}=\frac{v_{p}}{\sqrt{1+\varepsilon_{A, B}}},
$$

at which the dielectric and metal permittivities are opposite, so that SPPs with high $k$ can be excited:

$$
\varepsilon_{m}\left(v_{s p A}\right)=-\varepsilon_{A}, \quad \varepsilon_{m}\left(v_{s p B}\right)=-\varepsilon_{B} .
$$

In addition to SPPs, the structure supports volume plasmons (VPs) at the frequency determined by the condition $\varepsilon_{\|}=0$ and given by (for more details see [42])

$$
v_{\mathrm{vol}}=\frac{v_{p}}{\sqrt{1+\frac{1}{2}\left(\varepsilon_{A}+\varepsilon_{B}\right) \frac{d_{d}}{d_{m}}}} .
$$

Figures 3(b) and 3(c) correspond to the structures considered in Fig. 2, with two-dimensional maps showing the extended version of the dependence of the Purcell factor on frequency and $\varepsilon_{A, B}$ overlaid with the spectral position of the three modes SPP-A, SPP-B, and VP. We see that the peaks in the Purcell factor correspond to these identified plasmonic modes. The peak associated with the excitation of surface plasmon at the M-B interface (SPP-B) is always much stronger than the peaks for SPP-A and volume plasmon. This means that the emitter radiation goes primarily to the SPP-B mode, which is reasonable because for an emitter placed in a B layer, this mode is the easiest to couple into.

However, we also see features pertaining to the entire structure rather than just the immediate surroundings of the emitter. The most striking one is that the Purcell factor in the peak associated with SPP-B decreases once $\varepsilon_{A} \approx \varepsilon_{B}$ and greatly increases as the two SPP peaks become more separated from each other. We also see that the increase of the Purcell factor is markedly stronger when the SPP-B branch approaches the VP branch. This is most probably the effect of lower effective $\varepsilon_{\|}$, which results in a more extended isofrequency contour in the $k$ space, and consequently, in the increased photonic density of states [54]. However, this can also be interpreted as the interplay between surface and volume plasmons.

We can see the manifestation of the three modes in the Purcell spectra. For varying $\varepsilon_{B}$, the VP peak is discernible for $\varepsilon_{B}<\varepsilon_{A}$ at approximately $500-550 \mathrm{THz}$ for, e.g., $\varepsilon_{B}=2.2$ in Figs. 2(a) and 3(b). In contrast, this peak is not pronounced for $\varepsilon_{B}>\varepsilon_{A}$. For the SPP-A peak the behavior is opposite: there is no peak for $\varepsilon_{B}<\varepsilon_{A}$ (e.g., at $\varepsilon_{B}=2.2$ at $600 \mathrm{THz}$ ), but it appears for $\varepsilon_{B}>\varepsilon_{A}$. For the other case, i.e., when $\varepsilon_{A}$ is varied but 

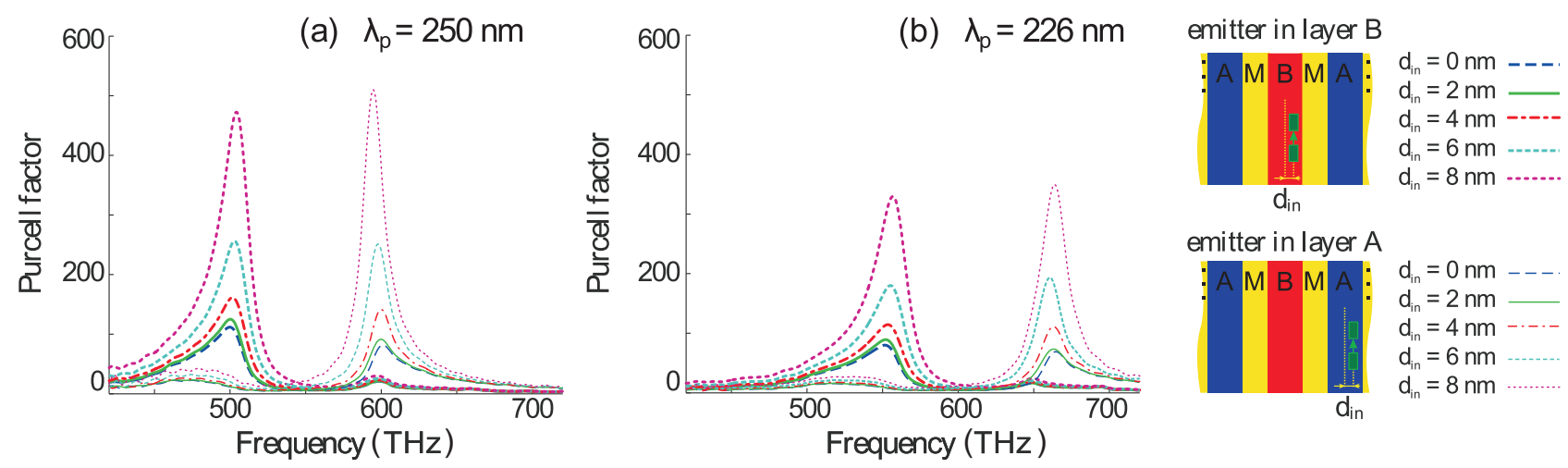

FIG. 4. Frequency dependence of the Purcell factor for a multilayer metamaterial structure [Fig. 1(a)] for different positions of point emitter in the central layer with $\varepsilon_{B}=4.6$ and $\varepsilon_{A}=3$ for different plasma frequencies of the metal layers: (a) $\lambda_{p}=250 \mathrm{~nm}$ and (b) $\lambda_{p}=226 \mathrm{~nm}$. The distance $d_{\text {in }}$ is the displacement of the emitter away from the center of the layer in which the emitter is placed (see insets).

the emitter is in layer $\mathrm{B}$, the behavior is essentially similar. Disappearing SPP-A- and VP-associated peaks for $\varepsilon_{A} \approx \varepsilon_{B}$ also confirms that the more these two SPP peaks are separated from each other, the higher the peak Purcell factor turns out to be.

In Fig. 4, we further investigate the effect of the emitter position in the structure on the spontaneous emission enhancement properties. To do so, we calculate the spectral dependencies of the Purcell factor for the different positions of the emitter, either across the central B layer or across the neighboring A layer in the same unit cell. (Since we consider the emitter to be a point dipole, we restrict ourselves to placing it in the dielectric layers to avoid singularities.) The emitter is shifted along the $z$ axis from the center of the respective layer by a varying distance $d_{\text {in }}$ (see inset in Fig. 4). We show the results for the biperiodic structures with two different values of $\lambda_{p}$ for the metal, and for $d_{\text {in }}$ varying from 0 to $8 \mathrm{~nm}$.

It can be seen that the Purcell factor increases dramatically when the emitter gets closer to the metallic layer, sometimes increasing from 280 to 560 with displacement of the emitter by as little as $2 \mathrm{~nm}$. One can also see that the type of layer where the emitter is embedded affects the frequency of the Purcell-factor peak in accordance with the observations in Figs. 2 and 3. Finally, by comparing Figs. 4(a) and 4(b) we can see that changing the properties of the metal also affects the spectral location of both peaks in the Purcell factor, again by virtue of changing the frequency of the surface plasmon polariton excitation at the nearest metal-dielectric interface.

It is important to distinguish the increase of the Purcell factor attributable to the coupling of emitted light to plasmonic excitations (radiative decay channel) and the well-known quenching effect, which is caused by nonradiative energy transfer and is considered as an undesirable energy loss. Both channels have complicated dependencies on the distance between the emitter and the metal surface, which are defined by electromagnetic fields, e.g., mode profile, light polarization, whether the emitter is an electric or a magnetic dipole, etc. Some simple cases were studied in the literature in detail. For instance, for an electric dipole close to a single metal-dielectric interface, the Purcell enhancement caused by plasmon emission exponentially depends on the emitter-metal distance. In contrast, in the quenching regime, the Purcell factor scales as $1 / a^{3}$, where $a$ is the characteristic distance between the emitter and the metal surface [49,57]. However, for a magnetic dipole close to a flat metal surface, the situation is different: the Purcell factor with a nonradiative decay depends as $1 / a$ on the distance to the surface [57]. In particular, it means that for a magnetic dipole, the radiative channel can dominate even for sub-10-nm distance where electric dipole radiation would be completely quenched [58]. For an electric dipole on top of a negative-index metamaterial waveguide with slow light, the scaling law $1 / a^{1.5}$ was empirically found [48].

Even more complex dependencies on structural parameters have been found out for the Purcell factor of an emitter in tiny metal-insulator-metal gaps [59]. The dominating effect among radiative and nonradiative decay channels is primarily defined by the distance between the emitter and the metal surface $h$; however, the spatial profile of the plasmon mode itself also plays a very important role. On the one hand, the quenching effect was shown to dominate when the emitter is at distances $h<5 \mathrm{~nm}$ to metal in a metal-dielectric-metal structure with dielectric layer thickness $H=30 \mathrm{~nm}$ [60]. On the other hand, it was experimentally demonstrated that once $H$ itself is below $5 \mathrm{~nm}$, plasmon emission dominates [51]. It appears that reducing the gap thickness leads to an increased mode confinement and emitter-mode overlap, which in turn enhances the emission of the plasmons sufficiently to exceed the quenching effect.

Furthermore, different field components have different distributions for the same mode, and consequently, the interplay between radiative and nonradiative decay channels strongly depends on dipole polarization [61]. It was theoretically shown that radiative decay dominates for the electric dipole which is perpendicular to the metal layers with an $H=8 \mathrm{~nm}$ gap and that the emission is mostly quenched for the dipole along the metal layer in the same structure.

We note that the goal of the present work is to investigate the influence of HMM multiscaling on the Purcell factor of the emitter inside it, and the multilayer structure is chosen as a model of possible realization. Thus, the electric dipole under consideration is oriented along metal layers in order to suppress the excitation of surface plasmons and focus on the properties of the structure as a metamaterial.

Since HMM includes many layers, it supports propagating modes that populate a broader region in the wave-vector 


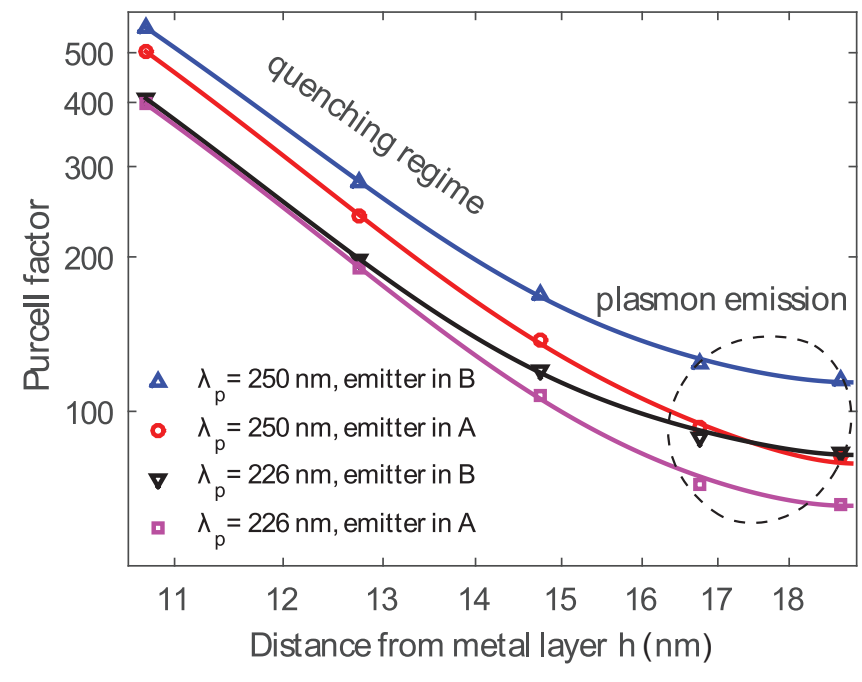

FIG. 5. Maximum value of the peak Purcell factor vs distance to the nearest metal layer $h$. (Simulation results are from Fig. 4.)

space than structures with only a few metal layers, such as a metal-insulator-metal structure. (In the limiting case, the number of layers is infinite and the resulting structure can have very different properties from the one containing a few layers.) Aside from high-propagation wave vectors, resonant bulk plasmon excitations in HMM are associated with slow energy propagation and an increase of Purcell factor because of it [48]. Finally, the field confinement is also very high in HMMs, so the question of whether plasmon coupling is stronger or weaker than quenching remains open. The answer may also nonmonotonically depend on the distance between the emitter and the closest metal layer. In Fig. 5, we plot the peak value of the Purcell factor as a function of $h=d_{A, B} / 2-d_{\text {in }}$, where $d=d_{A, B}$ is the thickness of the layer where the emitter is placed. We see that for small distances, the increase of the Purcell factor is steep, which means that quenching dominates for $h \lesssim 15 \mathrm{~nm}$. At the same time, for large distances $h \gtrsim 15 \mathrm{~nm}$, the dependence is rather flat, which indicates the change of the regime and plasmon emission.
This also confirms that our analysis presented in Figs. 2 and 3 was carried out in the regime where quenching should not be dominant, as the dipole was placed in the middle of the dielectric layer having $37.5 \mathrm{~nm}$ thickness.

\section{PURCELL EFFECT IN MULTIPERIODIC STRUCTURES WITH CAVITY LAYER}

In this section, we consider the structures with a cavity layer as shown in Fig. 1(b), where the emitter is placed in the cavity layer and the thickness of that layer $d_{C}$ is varied. In doing so, we keep the emitter at a fixed distance, $0.25 d_{B}=9.375 \mathrm{~nm}$, from the nearest metal-dielectric interface in order to keep constant the dominating effect of that interface identified in the previous section.

Figure 6(a) shows the spectral dependencies of the Purcell factor for several values of $d_{C}$ between 30 and $45 \mathrm{~nm}$ in structures with $\lambda_{p}$ equal to 250 and $226 \mathrm{~nm}$. We can see a general trend that the peak of the Purcell factor decreases when the cavity layer becomes thicker. The decrease is rather slight and achieves saturation above $d_{C}=40 \mathrm{~nm}$. This saturation character can be further confirmed by obtaining a continuous dependence of the Purcell factor on $d_{C}$ semianalytically by using a standard Green's function approach [9,62]. In Fig. 6(b), we see that nearly flat dependencies of the Purcell factor are observed for $d_{C}>1.5 d_{B}$.

This behavior confirms that the coupling between the emitter and the SPP mode at the nearest metal-dielectric interface is predominant; the dependence on $d_{C}$ is most likely caused by the presence of the second interface that moves closer to the emitter as $d_{C}$ decreases. The supposed influence of a localized bulk-plasmon mode present in the cavity layer [43] is not detected. The reason is that losses in metal strongly suppress hybridization of the SPP responsible for the bulk mode formation, as can be inferred from Fig. 3. Therefore, the losses lower the quality factor of the resonator formed by the cavity layer so much that it becomes insufficient for the formation of a Fabry-Pérot resonant mode. This is consistent with the observation of Ref. [43] that gain compensation of (a)

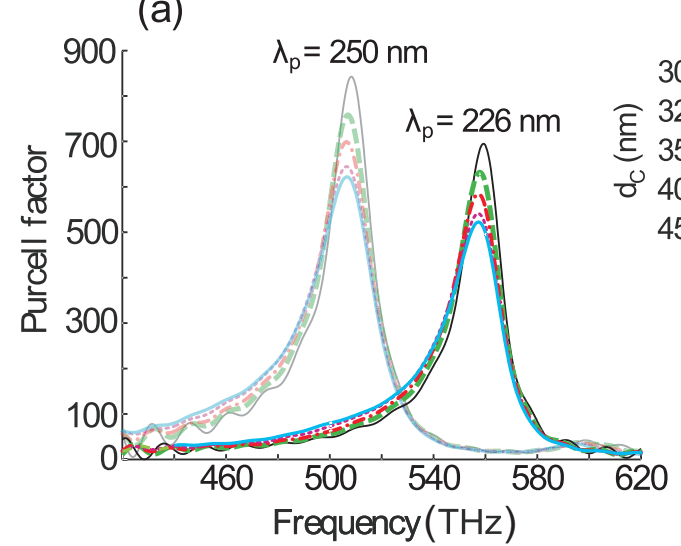

$250 \mathrm{~nm} 226 \mathrm{~nm}$

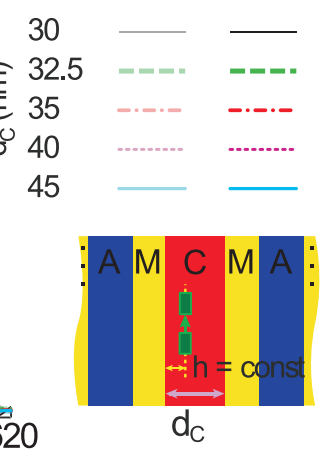

(b)

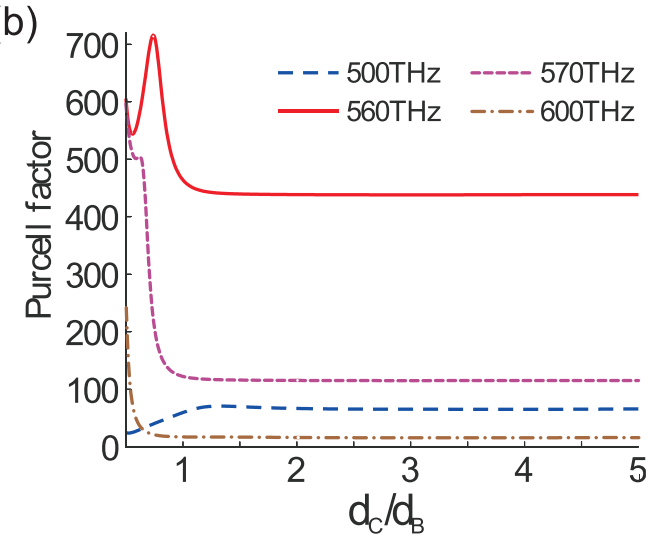

FIG. 6. (a) Dependence of the Purcell factor on frequency for two structures with cavity layer [Fig. 1(b)] for two different $\lambda_{p}$. The distance between the emitter and the metal layer is always kept at $h=0.25 d_{B}=9.375 \mathrm{~nm}$ (see inset). (b) The dependencies of Purcell factor on $d_{C} / d_{B}$ for $\lambda_{p}=226 \mathrm{~nm}$ at four characteristic dipole frequencies: to the left of the peak (500 THz), at the peak $(560 \mathrm{THz})$, to the right of the peak $(570 \mathrm{THz})$, and away from the peak $(600 \mathrm{THz})$ obtained by using standard Green's function approach $[9,62]$ for the structure with infinite $N$. 


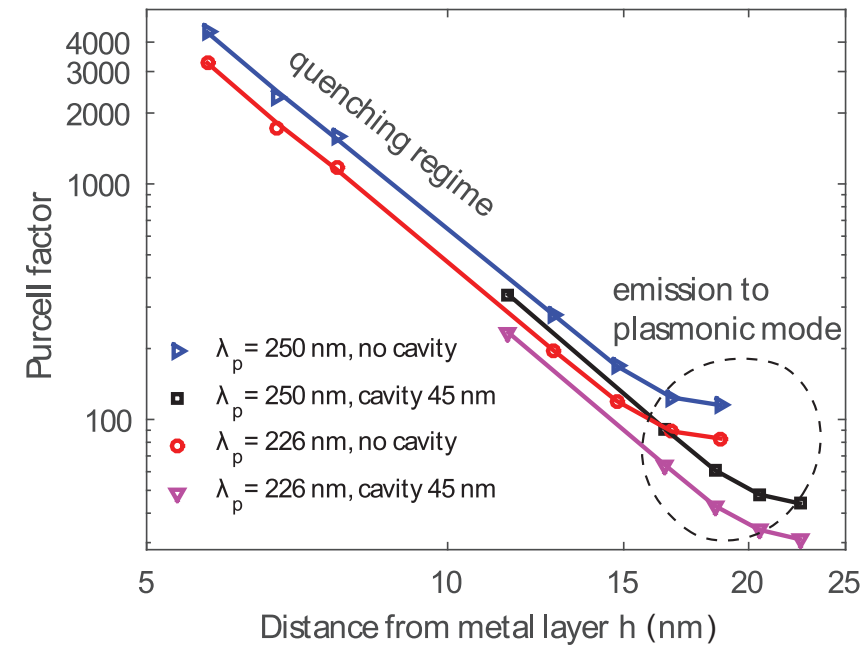

FIG. 7. Maximum value of Purcell factor vs distance to the closest metal layer $h$, defined as $h=d_{B}-d_{\text {in }}$ for regular structures and as $h=d_{C}-d_{\text {in }}$ for the structures with cavity $\left(d_{C}=45 \mathrm{~nm}\right)$. Marks show results of numerical simulations.

losses [63] was needed to observe the localized bulk mode in transmission-scenario experiments.

The dependence of the Purcell factor at a fixed frequency on $d_{C} / d_{B}$ in the range below 1.5, as shown in Fig. 6(b), can have different character depending on the choice of the frequency with respect to the location of the peak in Fig. 6(a). It can change from an increasing function for frequencies below the peak to a generally decreasing dependence for frequencies above the peak to a sharp drop at frequencies further away from the peak. At the peak frequency, the function has a more complicated nonmonotonic nature, having a maximum for $d_{C} \simeq 0.75 d_{B}$. These semianalytical calculations confirm the numerical simulation results of CST MICROWAVE STUDIO.

Finally, we compare the Purcell effect in biperiodic structures with and without a cavity layer for different cavity thickness $d_{C}$ and emitter placement within the cavity $d_{\text {in }}$. Similar to the previous case, we have calculated the maximum values of the Purcell factor and analyzed their dependence on the distance to the nearest metal boundary, $h$ (Fig. 7). As we can see, the Purcell factor in the structures with $d_{C}>d_{B}$ is less than in regular structures; this behavior persists for different $\lambda_{p}$ and is observed for all values of $d_{\text {in }}$ (see Fig. 7). On the other hand, the Purcell factor can be greatly increased in the structure with $d_{C}<d_{B}$ (Fig. 8). For the case $d_{C}=0.8 d_{B}$, which approximately corresponds to the maximum of the solid-line dependence in Fig. 6(b), the Purcell factor is seen to increase by a factor of 3-9, depending on $d_{\text {in }}$.

The comparison between regular and cavity-based biperiodic structures reveals that even though the cavity structures do not bring fundamentally new effects for our choice of parameters, the interplay between SPP excitations at different metal-dielectric boundaries can be significantly influenced by varying the cavity layer thickness. As a result, the enhancement of the Purcell effect stemming from the multiperiodicity (namely, from $\varepsilon_{B} \neq \varepsilon_{A}$, see Fig. 3) can be further controlled by the proper choice of the thickness of the layer containing the emitting center in question.
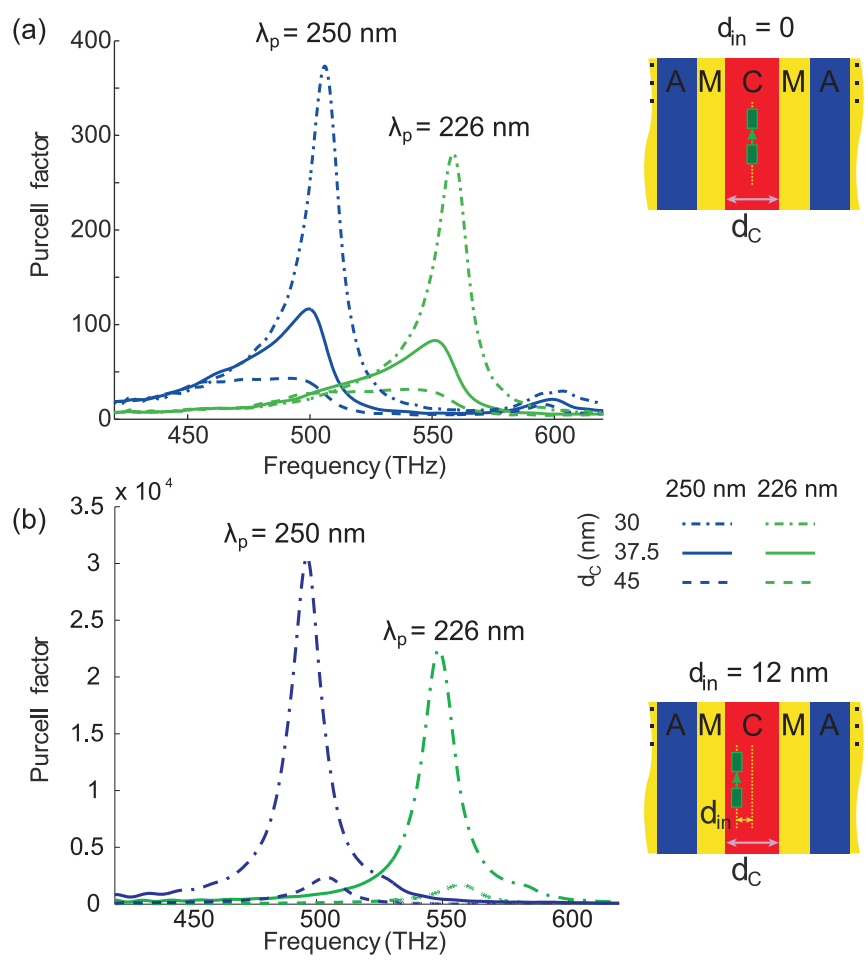

FIG. 8. Comparison of Purcell-factor dependencies on frequency for regular [Fig. 1(a)] and for structures with a cavity central layer [Fig. 1(b)] with $d_{C}=45$ and $30 \mathrm{~nm}$ for (a) $d_{\text {in }}=0$ (emitter at the cavity layer center) and (b) $d_{\text {in }}=12 \mathrm{~nm}$ (comparable to the case of Fig. 6).

\section{CONCLUSIONS}

We have theoretically studied the spontaneous emission enhancement (the Purcell effect) in biperiodic hyperboliclike metamaterials, i.e., periodic metal-dielectric multilayers where the odd-numbered dielectric layers have different dielectric permittivity from the even-numbered dielectric layers, resulting in a geometry that can be coded as $(\mathrm{MAMB})^{N}$, where $N$ is the number of periods. We have shown that the presence of multiperiodicity affects the spontaneous emission enhancement experienced by a point-dipole emitter placed in one of the dielectric layers, with the Purcell factors greater than in ordinary periodic hyperbolic metamaterial, and have characterized this additional enhancement with respect to the emitter placement and multilayer composition.

We have explicitly demonstrated that this Purcell-factor enhancement is primarily attributed to the surface excitation at the metal-dielectric interface closest to the emitter, which is the expected behavior for an emitter brought close to a metallic surface. Nevertheless, the degree of enhancement was found to be much stronger in multiperiodic structures where there are several distinct surface plasmon excitations compared to the regular periodic multilayers with only one kind of such SPP modes. Most likely, this behavior is due to the multiple-band character of the dispersion relation of the multiperiodic structures, so that a larger portion of the $k$ space becomes populated by propagating states that the emitter can couple with. Another, related increase of the Purcell factor was observed close to the region where the effective permittivity reaches near-zero values, again because the dispersion relation becomes more extended in $k$ space. 
We have also found out that structures with a "cavity" layer (i.e., with a layer whose thickness has been altered compared to the other layers of the same type) can bring about an additional Purcell-factor enhancement resulting from the interplay between surface plasmon excitations at the two metal-dielectric interfaces closest to the emitter.

We would like to point out that according to [47,51,52], one can further increase the Purcell factor by decreasing the dielectric and metal layer thicknesses and choosing metals with lower losses. Nevertheless, the goal of this paper is to study the effect of multiperiodicity of HMM rather than to engineer a structure with maximum Purcell factor, so we have focused on the general properties of the structure under consideration and did not perform optimization.

The combined action of all the described enhancement mechanisms can cause the Purcell factor to be more than an order of magnitude stronger compared to what one would expect by naively treating the multilayer in the effective medium approximation, i.e., without regard for its internal structure. Besides improving our understanding of light-matter interaction in complex plasmonic metamaterials, our results show that plasmonic multilayers with an internal structure that is only slightly more complex than a simple alternation of metal and dielectric layers can be used to tailor such properties as spontaneous emission of embedded atoms with high versatility.

\section{ACKNOWLEDGMENTS}

We are grateful to Alexander Poddubny and Yuri Kivshar for suggestions during manuscript preparation. One of us (S.V.Z.) wishes to acknowledge partial financial support from the People Programme (Marie Curie Actions) of the European Unions 7th Framework Programme FP7-PEOPLE2011-IIF under REA Grant Agreement No. 302009 (Project HyPHONE). This work was financially supported by the Government of the Russian Federation, Grant No. 074-U01, by the Ministry of Education and Science of the Russian Federation (Zadanie No. 3.1231.2014/K and Project No. 11.G34.31.0020, GOSZADANIE 2014/190, Zadanie No. 3.561.2014/K and No. 14.584.21.0009 10), by the Russian Foundation for Basic Research (Grants No. 14-02-31270 and No. 14-07-31272), and the Dynasty Foundation (Russia).
[1] H. N. S. Krishnamoorthy, Z. Jacob, E. Narimanov, I. Kretzschmar, and V. M. Menon, Science 336, 205 (2012).

[2] J. Luo, H. Chen, B. Hou, P. Xu, and Y. Lai, Plasmonics 8, 1095 (2013).

[3] J. Valentine, S. Zhang, T. Zentgraf, E. Ulin-Avila, D. A. Genov, G. Bartal, and Xiang Zhang, Nature 455, 376 (2008).

[4] E. Verhagen, R. de Waele, L. Kuipers, and A. Polman, Phys. Rev. Lett. 105, 223901 (2010).

[5] T. Xu, A. Agrawal, M. Abashin, K. J. Chau, and H. J. Lezec, Nature (London) 497, 470 (2013).

[6] M. Kuwata-Gonokami, N. Saito, Y. Ino, M. Kauranen, K. Jefimovs, T. Vallius, J. Turunen, and Y. Svirko, Phys. Rev. Lett. 95, 227401 (2005).

[7] G. Castaldi, V. Galdi, A. Alu, and N. Engheta, Phys. Rev. Lett. 108, 063902 (2012).

[8] Z. Jacob, I. I. Smolyaninov, and E. E. Narimanov, Appl. Phys. Lett. 100, 181105 (2012).

[9] I. Iorsh, A. Poddubny, A. Orlov, P. Belov, and Y. S. Kivshar, Phys. Lett. A 376, 185 (2012).

[10] A. V. Chebykin, A. A. Orlov, C. R. Simovski, Y. S. Kivshar, and P. A. Belov, Phys. Rev. B 86, 115420 (2012).

[11] A. Poddubny, I. Iorsh, P. Belov, and Y. Kivshar, Nat. Photonics 7, 948 (2013).

[12] D. R. Smith, D. Schurig, J. J. Mock, P. Kolinko, and P. Rye, Appl. Phys. Lett. 84, 13 (2004).

[13] D. Smith, P. Kolinko, and D. Schurig, J. Opt. Soc. Am. B 21, 1032 (2004).

[14] A. Degiron, D. Smith, J. Mock, B. Justice, and J. Gollub, Appl. Phys. A: Mater. Sci. Process. 87, 321 (2007).

[15] W. Yan, L. Shen, L. Ran, and J. A. Kong, J. Opt. Soc. Am. A 24, 530 (2007).
[16] V. P. Drachev, V. A. Podolskiy, and A. V. Kildishev, Opt. Express 21, 15048 (2013).

[17] Z. Jacob, L. V. Alekseyev, and E. Narimanov, Opt. Express 14, 8247 (2006).

[18] Z. Jacob, J.-Y. Kim, G. V. Naik, A. Boltasseva, E. E. Narimanov, and V. M. Shalaev, Appl. Phys. B: Lasers Opt. 100, 215 (2010).

[19] M. Y. Shalaginov, V. V. Vorobyov, J. Liu, M. Ferrera, A. V. Akimov, A. Lagutchev, A. N. Smolyaninov, V. V. Klimov, J. Irudayaraj, A. V. Kildishev et al., Laser Photonics Rev. 9, 120 (2015).

[20] J. K. Kitur, L. Gu, T. Tumkur, C. Bonner, and M. A. Noginov, ACS Photonics 2, 1019 (2015).

[21] K. L. Tsakmakidis, A. D. Boardman, and O. Hess, Nature (London) 450, 397 (2007).

[22] H. Hu, D. Ji, X. Zeng, K. Liu, and Q. Gan, Sci. Rep. 3, 1249 (2013).

[23] M. A. K. Othman, C. Guclu, and F. Capolino, Opt. Express 21, 7614 (2013).

[24] A. D. Neira, N. Olivier, M. E. Nasir, W. Dickson, G. A. Wurtz, and A. V. Zayats, Nat. Commun. 6, 7757 (2015).

[25] G. A. Wurtz, R. Pollard, W. Hendren, G. Wiederrecht, D. Gosztola, V. Podolskiy, and A. V. Zayats, Nat. Nanotechnol. 6, 107 (2011).

[26] C. Simovski, S. Maslovski, I. Nefedov, and S. Tretyakov, Opt. Express 21, 14988 (2013).

[27] S. Lang, M. Tschikin, S.-A. Biehs, A. Y. Petrov, and M. Eich, Appl. Phys. Lett. 104, 121903 (2014).

[28] X. Liu, T. Bright, and Z. Zhang, J. Heat Transfer 136, 092703 (2014).

[29] J. Yao, Z. Liu, Y. Liu, Y. Wang, C. Sun, G. Bartal, A. M. Stacy, and X. Zhang, Science 321, 930 (2008). 
[30] W. Dickson, G. A. Wurtz, P. Evans, D. O'Connor, R. Atkinson, R. Pollard, and A. V. Zayats, Phys. Rev. B 76, 115411 (2007).

[31] A. Kabashin, P. Evans, S. Pastkovsky, W. Hendren, G. Wurtz, R. Atkinson, R. Pollard, V. Podolskiy, and A. Zayats, Nat. Mater. 8, 867 (2009).

[32] N. Vasilantonakis, M. E. Nasir, W. Dickson, G. A. Wurtz, and A. V. Zayats, Laser Photonics Rev. 9, 345 (2015).

[33] M. A. Noginov, Y. A. Barnakov, G. Zhu, T. Tumkur, H. Li, and E. E. Narimanov, Appl. Phys. Lett. 94, 151105 (2009).

[34] M. A. Noginov, H. Li, Y. A. Barnakov, D. Dryden, G. Nataraj, G. Zhu, C. E. Bonner, M. Mayy, Z. Jacob, and E. E. Narimanov, Opt. Lett. 35, 1863 (2010).

[35] J. Kanungo and J. Schilling, Appl. Phys. Lett. 97, 021903 (2010).

[36] I. Avrutsky, I. Salakhutdinov, J. Elser, and V. Podolskiy, Phys. Rev. B 75, 241402 (2007).

[37] S. Ishii, A. V. Kildishev, E. Narimanov, V. M. Shalaev, and V. P. Drachev, Laser Photonics Rev. 7, 265 (2013).

[38] O. Kidwai, S. V. Zhukovsky, and J. E. Sipe, Phys. Rev. A 85, 053842 (2012).

[39] S. V. Zhukovsky, O. Kidwai, and J. E. Sipe, Opt. Express 21, 14982 (2013).

[40] A. V. Chebykin, A. A. Orlov, A. V. Vozianova, S. I. Maslovski, Y. S. Kivshar, and P. A. Belov, Phys. Rev. B 84, 115438 (2011).

[41] V. A. Podolskiy, P. Ginzburg, B. Wells, and A. V. Zayats, Faraday Discuss. 178, 61 (2015).

[42] A. A. Orlov, A. K. Krylova, S. V. Zhukovsky, V. E. Babicheva, and P. A. Belov, Phys. Rev. A 90, 013812 (2014).

[43] S. V. Zhukovsky, A. A. Orlov, V. E. Babicheva, A. V. Lavrinenko, and J. E. Sipe, Phys. Rev. A 90, 013801 (2014).

[44] V. N. Smolyaninova, B. Yost, D. Lahneman, E. E. Narimanov, and I. I. Smolyaninov, Sci. Rep. 4, 5706 (2014).

[45] E. E. Narimanov, Phys. Rev. X 4, 041014 (2014).

[46] S. V. Zhukovsky, S. V. Gaponenko, and A. V. Lavrinenko, in Optics of Aperiodic Structures-Fundamentals and Device Applications, edited by L. Dal Negro (Pan Stanford Publishing, Singapore, 2014).
[47] Y. C. Jun, R. D. Kekatpure, J. S. White, and M. L. Brongersma, Phys. Rev. B 78, 153111 (2008).

[48] P. Yao, C. Van Vlack, A. Reza, M. Patterson, M. M. Dignam, and S. Hughes, Phys. Rev. B 80, 195106 (2009).

[49] G. Ford and W. Weber, Phys. Rep. 113, 195 (1984).

[50] A. Kinkhabwala, Z. Yu, S. Fan, Y. Avlasevich, K. Müllen, and W. Moerner, Nat. Photonics 3, 654 (2009).

[51] R. R. Chance, A. Prock, and R. Silbey, in Advances in Chemical Physics, Vol. 37, edited by I. Prigogine and Stuart A. Rice (John Wiley \& Sons, Inc., Hoboken, NJ, 1978).

[52] G. M. Akselrod, C. Argyropoulos, T. B. Hoang, C. Ciracì, C. Fang, J. Huang, D. R. Smith, and M. H. Mikkelsen, Nat. Photonics 8, 835 (2014).

[53] A. A. Orlov, I. V. Iorsh, S. V. Zhukovsky, and P. A. Belov, Photonics Nanostruct. 12, 213 (2014).

[54] A. V. Chebykin, A. A. Orlov, A. S. Shalin, A. N. Poddubny, and P. A. Belov, Phys. Rev. B 91, 205126 (2015).

[55] A. E. Krasnok, D. S. Filonov, C. R. Simovski, Y. S. Kivshar, and P. A. Belov, Appl. Phys. Lett. 104, 133502 (2014).

[56] A. E. Krasnok, A. P. Slobozhanyuk, C. R. Simovski, S. A. Tretyakov, A. N. Poddubny, A. E. Miroshnichenko, Y. S. Kivshar, and P. A. Belov, Sci. Rep. 5, 12956 (2015).

[57] R. Chance, A. Prock, and R. Silbey, Adv. Chem. Phys. 37, 1 (1978)

[58] D. N. Chigrin, D. Kumar, D. Cuma, and G. von Plessen, ACS Photonics 3, 27 (2016).

[59] R. Faggiani, J. Yang, and P. Lalanne, ACS Photonics 2, 1739 (2015)

[60] C. Belacel, B. Habert, F. Bigourdan, F. Marquier, J.-P. Hugonin, S. M. de Vasconcellos, X. Lafosse, L. Coolen, C. Schwob, C. Javaux et al., Nano Lett. 13, 1516 (2013).

[61] J. Yang, R. Faggiani, and P. Lalanne, Nanoscale Horiz. 1, 11 (2016).

[62] M. S. Tomas and Z. Lenac, Phys. Rev. A 60, 2431 (1999).

[63] X. Ni, S. Ishii, M. D. Thoreson, V. M. Shalaev, S. Han, S. Lee, and A. V. Kildishev, Opt. Express 19, 25242 (2011). 